\title{
Mini-Invasive floating metatarsal osteotomy for resistant or recurrent neuropathic plantar metatarsal head ulcers
}

\author{
Eran Tamir ${ }^{1,2+}$, Aharon S. Finestone ${ }^{1,2^{*}+}$, Erez Avisar $^{1}$ and Gabriel Agar ${ }^{1}$
}

\begin{abstract}
Background: Patients with peripheral neuropathy and pressure under a relatively plantar deviated metatarsal head frequently develop plantar foot ulcers. When conservative management with orthotics and shoes does not cure the ulcer, surgical metatarsal osteotomy may be indicated to relieve the pressure and enable the ulcer to heal. The purpose of this study is to evaluate the use of a mini-invasive floating metatarsal osteotomy in treating recalcitrant ulcers or recurrent ulcers plantar to the metatarsal heads in patients with diabetes mellitus (DM) related neuropathy.

Methods: Computerized medical files of patients with diabetic neuropathy treated with an osteotomy during 2013 and 2014 were retrospectively reviewed. There were 20 osteotomies performed on 17 patients (mean age 58 years). The patients had a diagnosis of DM for a mean of 17 years. All ulcers were University of Texas grade 1A; mean ulcer age was 19 months.

Results: After 17/20 operations, the ulcer completely resolved after 6 weeks and did not recur after a mean follow-up of 11.5 months. One patient developed an early post-operative infection with osteomyelitis at the osteotomy site (proximal shaft of the fifth metatarsal) that needed debridement and IV antibiotics. In the other 19 cases, the surgical wound healed within 1 week. Asymptomatic radiological non-union developed in six cases (30 \%).
\end{abstract}

Conclusions: Mini-invasive floating metatarsal osteotomy can cure resistant and recurrent University of Texas grade $1 \mathrm{~A}$ ulcerations plantar to the metatarsal heads in neuropathic patients.

Keywords: Diabetic foot, Prophylactic surgery, Metatarsal osteotomy, Amputation

\section{Background}

Pressure ulcers are common complications in patients with peripheral neuropathy. Most peripheral neuropathy is related to diabetes mellitus (DM). The annual incidence of ulcers in patients with DM is about $2 \%$ [1], and they have been implicated as a causative factor in up to $84 \%$ of diabetic foot amputations [2].

In the presence of sensory neuropathy and lack of protective sensation, an ulcer can develop in a foot with normal anatomy as result of an acute injury. More often, however, an ulcer develops when an anatomic abnormality of the foot is present that results in increased local

\footnotetext{
*Correspondence: asff@inter.net.il

${ }^{\dagger}$ Equal contributors

${ }^{1}$ Department of Orthopaedic Surgery, Assaf HaRofeh Medical Center, Zerrifin, Affiliated to the Sackler School of Medicine, Tel Aviv University, Tel Aviv, Israel

${ }^{2}$ Maccabi Health Services, POB 1424, Reut 71799, Israel
}

pressure. A frequent site of ulcers is under the heads of the metatarsals [3], where a flexion deformity (relative to the other metatarsals) results in elevated local pressure [4].

Primary off-loading with a cast is usually an effective treatment to achieve ulcer closure [5-7]. Afterwards, continued care is given with special shoes and orthotics to prevent recurrence. But these conservative methods sometimes fail due to complications or lack of compliance [8]. Surgical off-loading may then be indicated $[9,10]$. The purpose of the operation is to decrease the pressure plantar to the affected metatarsal head. This can be achieved by several surgical techniques including plantar condylectomy, Weil osteotomy with metal fixation, closing wedge metatarsal osteotomy with metal fixation, and Helal osteotomy [11-15]. While all these open methods are effective in off-loading, they tend to have relatively high 
complication rates because of wound dehiscence and/or post-operative infection. The mini-invasive floating metatarsal osteotomy is an effective off-loading procedure with a low complication rate because soft tissue damage is minimal and metal fixation is not used. The purpose of this retrospective study is to assess the post-operative results of this treatment modality.

\section{Methods}

We performed a retrospective chart review on all patients with DM who had a minimally invasive floating metatarsal osteotomy for the treatment of recalcitrant or recurrent neuropathic plantar metatarsal head ulcers between 2013 and 2014 (inclusion criteria). Patients were identified by ICD codes and operating theater records. All patients were operated on by a single surgeon (ET). No patients were excluded. Parameters collected included demographics, cause of neuropathy, type and duration of DM, recent HbA1c levels, duration and location of current ulcer, location of osteotomy (metatarsal neck or shaft), ulcer status 6 weeks after surgery, and complications. Major complications included any infection necessitating antibiotic treatment. Minor complications were defined as any other adverse event including recurrence, a transfer lesion, and non-union. The study was approved by the Assaf Harofeh Medical Center IRB.

\section{Operative technique and post-operative management}

Antibiotics (2 gr IV cephalosporin) were given preoperatively. Anesthesia by ankle block was performed with $15 \mathrm{~cm}^{3}$ of $1 \%$ lidocaine except in patients with neuropathy severe enough to make anesthesia unnecessary. A 3-mm incision was made dorsally at the planned osteotomy site after fluoroscopic identification. The bone was exposed by blunt dissection with a mosquito. A perpendicular or short oblique osteotomy was made at the neck or diaphysis of the affected metatarsus with a $12 \times 2 \mathrm{~mm}$ Shannon burr at a speed of 1600 RPM and a torque of $80 \mathrm{Nm}$ (Figs. 1, 2, and 3). Fluoroscopy was used again to confirm completion of the osteotomy. Following the osteotomy, the metatarsal head was displaced dorsally. Skin closure was achieved with a single 4-0 nylon suture. Full weight-bearing in a postoperative shoe was permitted immediately.

\section{Results}

Twenty procedures were performed in 17 patients; all but one were males. Their mean age was 58 (median 57, range 42 to 75 , IQR 56 to 63 ) years. All patients were diagnosed with late-onset DM for a mean and median of 17 (range 4 to 39 , IQR 13 to 20 ) years and their mean HbA1c was 8.1 (median 7.1, range 4.9 to 12.2, IQR 6.5 to 10.5$) \mathrm{g} / \mathrm{dL}$. One patient was post-pancreas transplantation. At the time of surgery, 11/17 patients were already being treated with insulin.

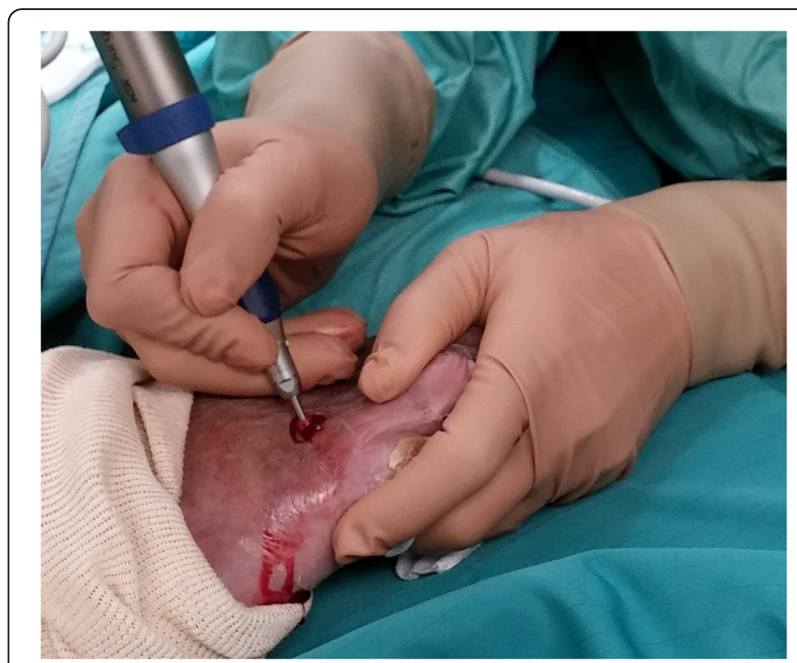

Fig. 1 Surgical procedure for metatarsal osteotomy using a $12 \times 2 \mathrm{~mm}$ Shannon burr

All patients had adequate blood supply with palpable pedal pulses or ABI index above 0.7. All ulcers were classified as University of Texas 1A (no infection, no ischemia, ulcer not penetrating to deep structures) [16] and the ulcer's mean age was 19 (median 11, range 1 to

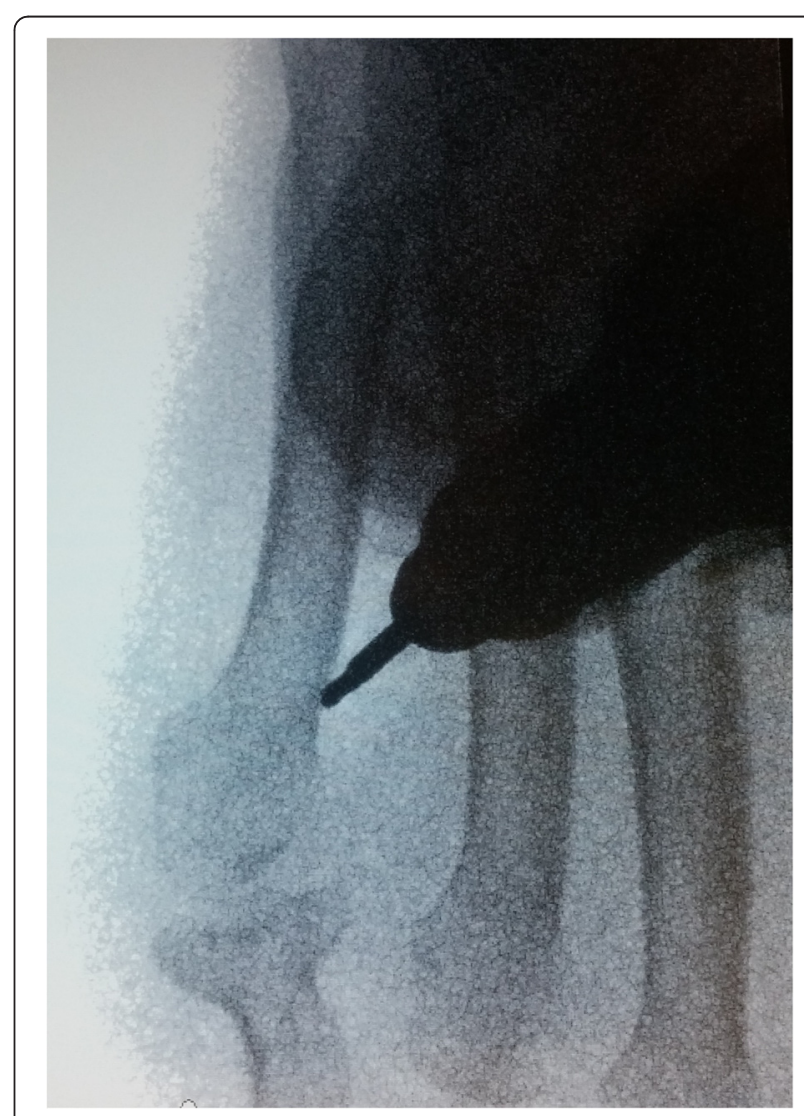

Fig. 2 Surgical procedure for metatarsal osteotomy using Shannon burr, fluoroscopic view 


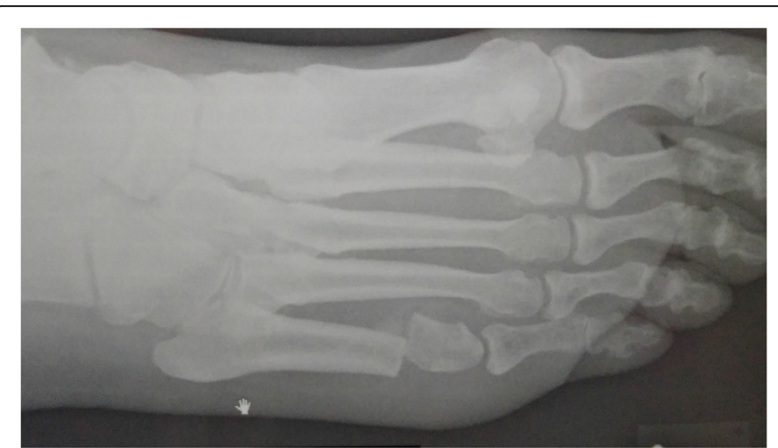

Fig. 3 Oblique X-ray of patient in following figures, 1 month following surgery. The fifth MT head is significantly elevated and the osteotomy is healing

60, IQR 4 to 36 ) months. Of 18 primary procedures, in 15 the floating osteotomy included 1 metatarsal and in 3 the osteotomy included 2 metatarsals (the additional metatarsal was always the fourth, secondary to the third). Case details are presented in Table 1.

One patient had a simultaneous bilateral procedure and two patients had second procedures due to the development of transfer ulcers. With the exception of one case, all surgical wounds (95\%) healed within 1 week. In that case, an early post-operative infection with osteomyelitis developed at the osteotomy site (proximal shaft of the fifth metatarsal). The patient was successfully treated by hospital

Table 1 Details of osteotomies and results

\begin{tabular}{lll}
\hline & Variable & Value (\%) \\
\hline Metatarsal & 2 & $5(25 \%)$ \\
& 3 & $4(20 \%)$ \\
& 3 and 4 & $3(15 \%)$ \\
Side & 4 & $8(40 \%)$ \\
& Right & \\
Osteotomy location & Left & $14(70 \%)$ \\
& Neck & $6(30 \%)$ \\
Healed & Shaft & $12(60 \%)$ \\
& Yes & $8(40 \%)$ \\
Total & No & $17(85 \%)$ \\
& & $3(15 \%)$ \\
& Yes & $14(70 \%)$ \\
& No & $6(30 \%)$ \\
& & $20(100 \%)$ \\
& &
\end{tabular}

admission with bone debridement and IV antibiotics per culture.

After 17/20 operations, the ulcer completely resolved after 6 weeks (no callus plantar to the affected metatarsal head) and did not recur after a mean follow-up of 11.5 (median 10, range 3 to 21 , IQR 8 to 16 ) months. The remaining three patients showed improvement but were not cured.

Non-union (lack of solid bone formation across the osteotomy site 6 months after the procedure) developed in six cases (30\%), three after osteotomy of the neck and three after osteotomy of the shaft. All patients with non-union were asymptomatic. Two patients developed a transfer lesion, one below the fourth metatarsal head 5 months after an osteotomy of the second metatarsal neck and the other below the second metatarsal head, 10 months after osteotomy of the third metatarsal neck.

\section{Discussion}

Using mini-invasive floating metatarsal osteotomy, we attained an $85 \%$ cure rate in the treatment of University of Texas grade 1A persistent or recurrent foot ulcers. In the long run, these ulcers are known to carry considerable morbidity in patients with DM and have been implicated as a causative factor for infection, hospitalization, amputations, and death.

Surgery correcting foot deformities, thereby decreasing the plantar pressure (internal off-loading), is indicated when conservative methods fail (usually after at least 6 weeks of off-loading with orthotic treatment). This failure might be related to complications of the off-loading, lack of compliance, persistence of pressure below a metatarsal head, or recurrent ulceration despite the off-loading treatment with shoes and orthotics. Contraindications for surgery include ischemia, infection, and ulcers penetrating deep structures.

Surgical options for recurrent or recalcitrant ulcers include Achilles tendon lengthening, [17] metatarsal osteotomies, debridement to metatarsal head resection, and limited amputations [18-20]. There are very few references relating to metatarsal osteotomies for off-loadingresistant and recurrent metatarsal plantar ulcers. Fleischli et al. reported 20 diabetic patients who underwent 22 open dorsiflexion metatarsal base osteotomies with internal fixation for treatment of chronic persistent or recurrent neuropathic forefoot ulcers [21]. Complete ulcer healing was noted in 21 cases (95\%). Complications occurred in 15 cases (68\%) including acute Charcot disease (32\%), deep wound infections (14\%), and transfer lesions (9\%). There were no cases of ulcer recurrence.

Tilo reported on 52 patients who had metatarsal osteotomies without internal fixation for the treatment of chronic neuropathic ulcerations [13]. The most common osteotomy used was the osteoclasis type, where a short 


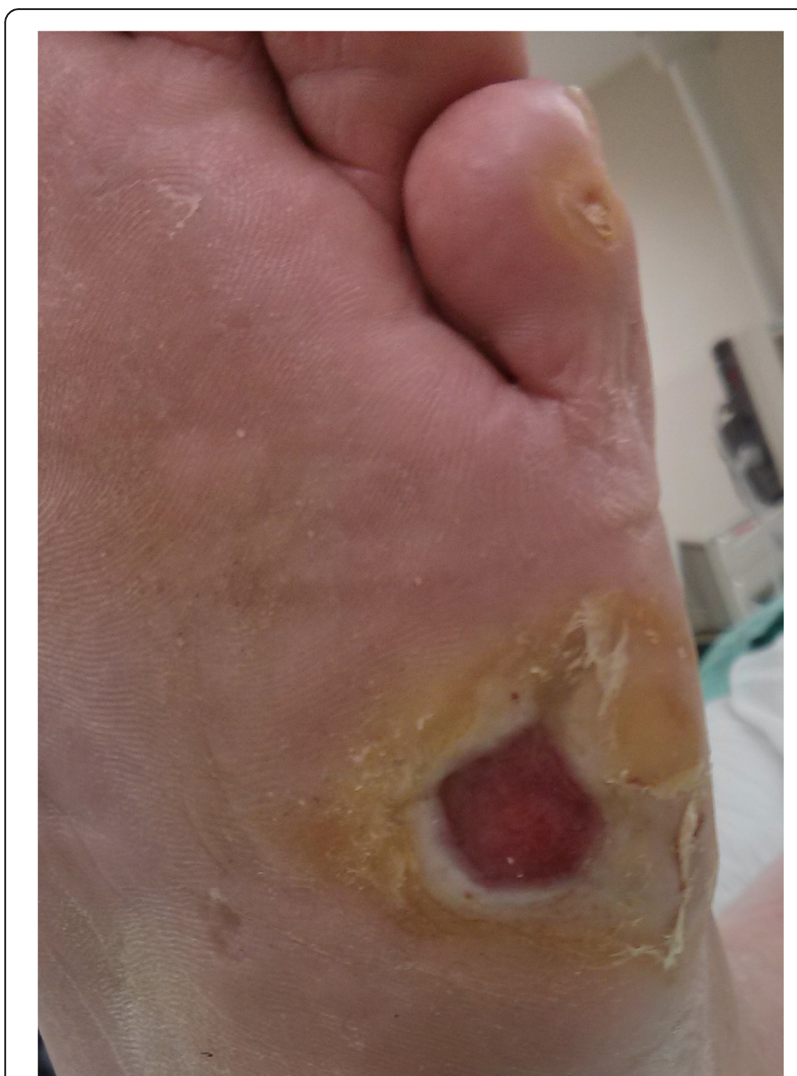

Fig. 4 Pre-operative view of chronic recurrent ulcer under the fifth MT head

(partial) osteotomy was made dorsal to the metatarsal neck, part of the neck was removed with a rongeur and the neck fractured by pushing the head dorsally. No early post-operative complications were noted. Reulceration developed in $6 \%$ and transfer ulcers developed in $26.5 \%$ of the cases 17 months following the procedure.

Other types of open osteotomies include the Weil osteotomy and a dorsal closing wedge osteotomy with internal fixation with plate and screws. While these procedures are effective in off-loading the ulcer, the post-operative complication rates of infection and wound dehiscence are relatively high.

Minimally invasive foot surgery has become quite popular among foot surgeons during the last decade, due to safer methods that use especially designed drills with low speed and high torque to prevent damage to nerves and blood vessels. Applying a minimally invasive technique to metatarsal osteotomies offers effective off-loading with a very low complication rate due to the minimal soft tissue damage. Moreover, the patient is permitted full weight-bearing in a post-operative shoe making the post-operative period easier for the patient. No internal fixation is used (floating osteotomy) and the affected metatarsal head is elevated by the weight-bearing and "settles" in its new position (Fig. 3). The plantar soft tissue reacts rapidly to the off-loading with healing of the ulcer, elimination of the callus, and reappearance of normal skin at the affected area (Figs. 4, 5, and 6).

The non-union rate was quite high (30\%) in this study, both at the neck and the diaphysis, but was asymptomatic in all patients and without clinical sequela. There were no cases of acute Charcot osteo-arthropathy. Two patients developed a transfer lesion 4 and 10 months after the procedure, which were successfully treated with another osteotomy. In three cases, the osteotomy failed to off-load the metatarsal head. One of these was a fourth metatarsal neck osteotomy performed on a foot following an open fifth ray resection, in a morbidly obese patient. Although the post-operative X-ray demonstrated significant elevation of the fourth metatarsal head, the ulcer only decreased in size but did not completely heal after 6 weeks. In the other two cases, the metatarsal head could not be elevated intra-operatively when pushed upwards after a complete osteotomy was done, probably due to fibrosis of the inter-metatarsal ligaments and other soft tissues.

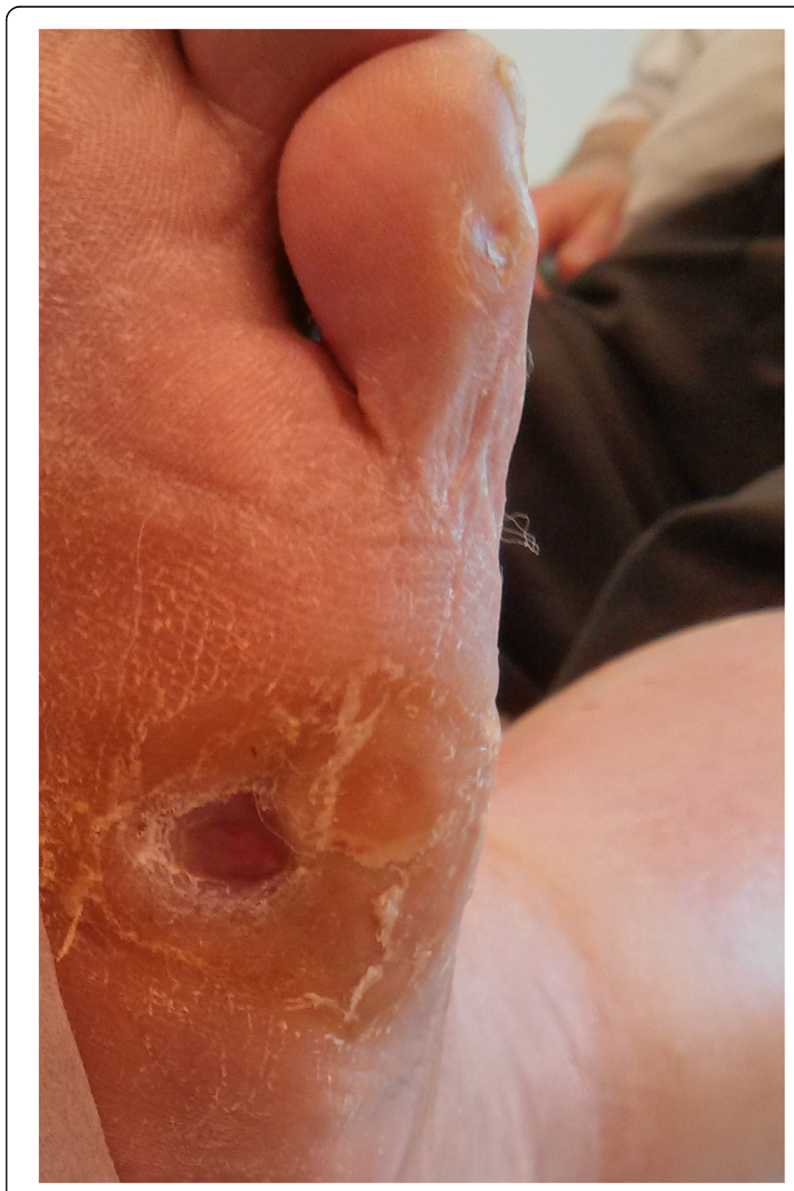

Fig. 5 Ulcer, 6 days after surgery. The ulcer is healing rapidly while the patient is fully weight-bearing 


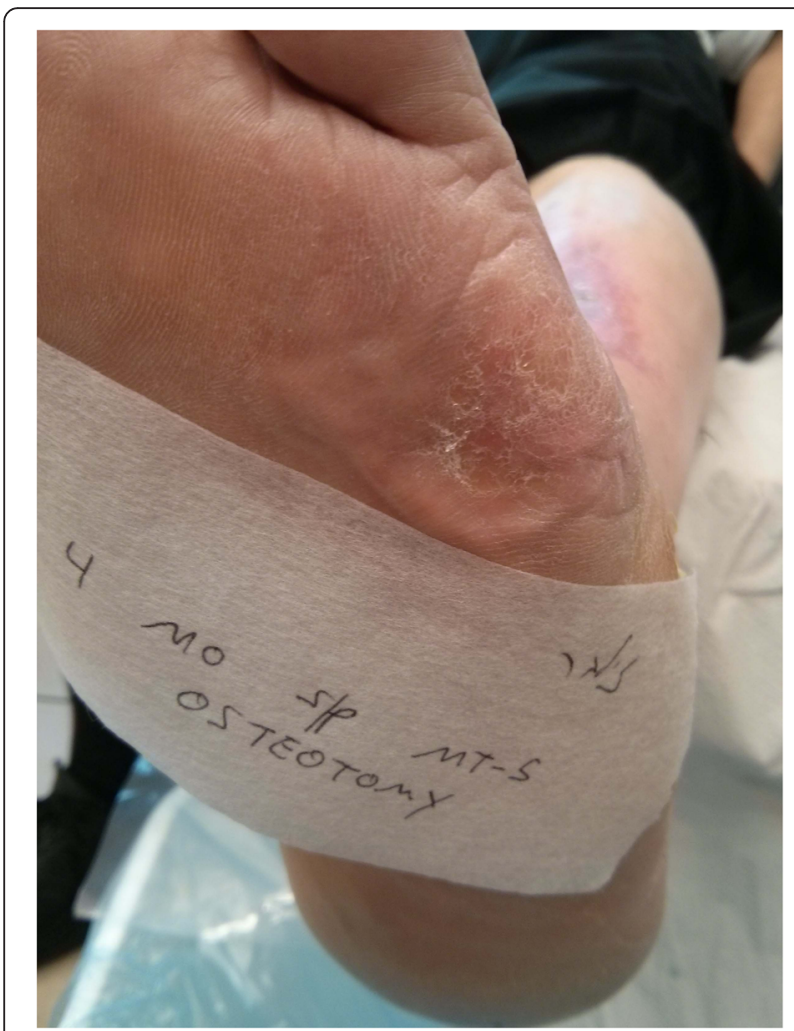

Fig. 6 Ulcer site, 4 months after surgery. The skin under the fifth MT head is almost normal

There was one post-operative infection at the osteotomy site. This osteotomy was performed at the proximal diaphysis of a fifth metatarsus for an ulcer under the head in a morbidly obese patient. The patient was not compliant and returned to work 2 days following surgery. He returned 2 weeks later with a purulent discharge from the osteotomy site. He was admitted to hospital for bone debridement and culture-directed antibiotic treatment. Eventually, he recovered with a non-union. On review of this case, we concluded that the reason for this complication was probably soft tissue damage at the osteotomy site caused by the movements of the fifth metatarsal and the long lever arm. Therefore, it is probably safer to perform a fifth metatarsal osteotomy at the neck and not in the diaphysis.

\section{Conclusions}

Mini-invasive floating metatarsal osteotomy is a safe and effective curative and prophylactic procedure for resistant and recurrent University of Texas grade 1 neuropathic plantar metatarsal head ulcers. Teams treating patients with complications of diabetes should include clinicians able to perform advanced curative and prophylactic surgery where appropriate.

\section{Abbreviations}

DM, diabetes mellitus; HbA1c, hemoglobin A1c; ICD, international classification of diseases; IRB, institutional review board; IQR, interquartile range; IV, intravenous; MT, metatarsal; RPM, rounds per minute

\author{
Acknowledgements \\ None \\ Funding \\ No funding was received from any party relating to this study.
}

Availability of data and materials

Raw unidentified data are available at request from the corresponding author.

\section{Authors' contributions}

ET, ASF, and GA conceived and designed the study. ET collected the data, ASF and EA analyzed the data, ET and EA drafted the manuscript, and GA supervised the whole study. The contribution of ET and ASF was equal. All authors read and approved the final manuscript.

\section{Competing interests}

The authors declare that they have no competing interests.

\section{Consent for publication}

Patients gave written consent that their clinical photographs be published so long as no identifiable material was presented.

\section{Ethics approval and consent to participate}

Assaf Harofeh MC, 0159-15-ASF. Consent is not required by IRB.

\section{Level of evidence}

IV-case series without control.

Received: 2 March 2016 Accepted: 25 June 2016

Published online: 11 July 2016

\section{References}

1. Ramsey SD, Newton K, Blough D, McCulloch DK, Sandhu N, Reiber GE, Wagner EH. Incidence, outcomes, and cost of foot ulcers in patients with diabetes. Diabetes Care. 1999;22(3):382-7.

2. Pecoraro RE, Reiber GE, Burgess EM. Pathways to diabetic limb amputation. Basis for prevention. Diabetes Care. 1990;13(5):513-21.

3. Cowley MS, Boyko EJ, Shofer JB, Ahroni JH, Ledoux WR. Foot ulcer risk and location in relation to prospective clinical assessment of foot shape and mobility among persons with diabetes. Diabetes Res Clin Pract. 2008;82(2):226-32.

4. Ledoux WR, Shofer JB, Cowley MS, Ahroni JH, Cohen V, Boyko EJ. Diabetic foot ulcer incidence in relation to plantar pressure magnitude and measurement location. J Diabetes Complications. 2013;27(6):621-6.

5. Myerson M, Papa J, Eaton K, Wilson K. The total-contact cast for management of neuropathic plantar ulceration of the foot. J Bone Joint Surg Am. 1992;74(2):261-9.

6. Bus SA, van Deursen RW, Armstrong DG, Lewis JE, Caravaggi CF, Cavanagh PR, International Working Group on the Diabetic Foot. Footwear and offloading interventions to prevent and heal foot ulcers and reduce plantar pressure in patients with diabetes: a systematic review. Diabetes Metab Res Rev. 2016:32 Suppl 1:99-118.

7. Bus SA, van Netten JJ, Lavery LA, Monteiro-Soares M, Rasmussen A, Jubiz Y, Price PE, International Working Group on the Diabetic Foot. IWGDF guidance on the prevention of foot ulcers in at-risk patients with diabetes. Diabetes Metab Res Rev. 2016;32 Suppl 1:16-24.

8. Armstrong DG, Lavery LA, Kimbriel HR, Nixon BP, Boulton AJ. Activity patterns of patients with diabetic foot ulceration: patients with active ulceration may not adhere to a standard pressure off-loading regimen. Diabetes Care. 2003;26(9):2595-7.

9. Tamir E. Treating the diabetic ulcer: practical approach and general concepts. Isr Med Assoc J. 2007;9(8):610-5.

10. Armstrong DG, Frykberg RG. Classifying diabetic foot surgery: toward a rational definition. Diabet Med. 2003;20(4):329-31. 
11. Martin WJ, Weil LS, Smith SD. Surgical management of neurotrophic ulcers in the diabetic foot. J Am Podiatry Assoc. 1975;65(4):365-73.

12. Singer A. Surgical treatment of mal perforans. Arch Surg. 1976;111(9):964-8.

13. Tillo TH, Giurini JM, Habershaw GM, Chrzan JS, Rowbotham JL. Review of metatarsal osteotomies for the treatment of neuropathic ulcerations. J Am Podiatr Med Assoc. 1990;80(4):211-7.

14. Addante JB. Metatarsal osteotomy as an office procedure to eradicate intractable plantar keratosis. J Am Podiatry Assoc. 1970;60(10):397-9.

15. Helal B. Metatarsal osteotomy for metatarsalgia. J Bone Joint Surg Br. 1975;57(2):187-92.

16. Lavery LA, Armstrong DG, Harkless LB. Classification of diabetic foot wounds. J Foot Ankle Surg. 1996;35(6):528-31.

17. Colen LB, Kim CJ, Grant WP, Yeh JT, Hind B. Achilles tendon lengthening: friend or foe in the diabetic foot? Plast Reconstr Surg. 2013;131(1):37e-43e.

18. Armstrong DG, Rosales MA, Gashi A. Efficacy of fifth metatarsal head resection for treatment of chronic diabetic foot ulceration. J Am Podiatr Med Assoc. 2005;95(4):353-6.

19. Aragon-Sanchez J, Lazaro-Martinez UL, Hernandez-Herrero C, Campillo-Vilorio N, Quintana-Marrero Y, Garcia-Morales E, Hernandez-Herrero MJ. Does osteomyelitis in the feet of patients with diabetes really recur after surgical treatment? Natural history of a surgical series. Diabet Med. 2012;29(6):813-8.

20. Dalla Paola L, Faglia E, Caminiti M, Clerici G, Ninkovic S, Deanesi V. Ulcer recurrence following first ray amputation in diabetic patients: a cohort prospective study. Diabetes Care. 2003;26(6):1874-8.

21. Fleischli JE, Anderson RB, Davis WH. Dorsiflexion metatarsal osteotomy for treatment of recalcitrant diabetic neuropathic ulcers. Foot Ankle Int. 1999; 20(2):80-5.

\section{Submit your next manuscript to BioMed Central and we will help you at every step:}

- We accept pre-submission inquiries

- Our selector tool helps you to find the most relevant journal

- We provide round the clock customer support

- Convenient online submission

- Thorough peer review

- Inclusion in PubMed and all major indexing services

- Maximum visibility for your research

Submit your manuscript at www.biomedcentral.com/submit

C Biomed Central 